\title{
Influence of Radiation on Magneto-hydrodynamics Flow Over an Exponentially Stretching Sheet Embedded in a Thermally Stratified Porous Medium in the Presence of Heat Source
}

\author{
Isaac Ogechi Senge ${ }^{1}$, Emmanuel Olubayo Oghre ${ }^{2}$ and Idongesit Fred Ekang ${ }^{3}$ \\ ${ }^{1}$ Department of Mathematics, Faculty of Physical Science, University of Benin, Benin City, Nigeria \\ e-mail: Sengs4mail@yahoo.com \\ ${ }^{2}$ Department of Mathematics, Faculty of Physical Science, University of Benin, Benin City, Nigeria \\ e-mail: eoghre@yahoo.com \\ ${ }^{3}$ Department of Mathematics, Faculty of Science, University of Uyo, Uyo, Nigeria \\ e-mail: idongesitekang@uniuyo.edu.ng
}

\begin{abstract}
The influence of radiation on magneto-hydrodynamics (MHD) boundary layer flow over an exponentially stretching sheet embedded in a thermally stratified porous medium in the presence of heat source and suction/blowing was investigated.

Similarity transformation was used to convert the governing equations from partial differential equations into a system of non-linear ordinary differential equations. Solving numerically, we used shooting method along with fourth order Runge-Kutta technique to obtained numerical values.

The effects of the obtained numerical values of the dimensionless parameters on skinfriction coefficient, Nusselt number, velocity profile and temperature profile are illustrated in table and graphs plotted using MATLAB. Comparison of the velocity profile with previously published work was presented and found to be in good agreement.
\end{abstract}

\section{Introduction}

The present century saw a thorough and better understanding of the principles of fluid mechanics and knowledge of how to apply them to any real life problems. The interest on MHD flow over a stretching sheet have witnessed tremendous growth in the

Received: August 10, 2020; Accepted: September 13, 2020

2010 Mathematics Subject Classification: 76.

Keywords and phrases: radiation, MHD, thermally stratified porous medium.

Copyright () 2021 the Authors 
past few years based on its numerous engineering and industrial applications such as production of sheeting materials (metals and polymer sheets), paper production, metal spinning, the boundary layer along a liquid film and design of the plastic films.

The flow of a viscous, incompressible fluid over a stretching sheet was first considered by Sakiadis [1], who wrote on boundary layer behavior on continuous solid surface moving with a constant velocity. Extending the problem of Sakiadis [1], by solving analytically, Crane [2] worked on two dimensional flows over a stretching sheet. Gupta and Gupta [3] investigated the heat and mass transfer in the flow over a stretching surface (with suction/blowing) issuing from a thin slit. Elbashbeshy [4] brought a different dimension with heat transfer over an exponentially stretching continuous surface with suction. Bidin and Nazar [5] focused on the effect of thermal radiation on the steady laminar two dimensional boundary layer flow and heat transfer over an exponentially stretching sheet, which has been solved analytically by Sajid and Hayat [6]. Idowu and Usman [7] analyzed the influence of thermal radiation on MHD boundary layer flow of a viscous fluid over an exponentially stretching sheet which they solved analytically. Nadeem et al. [8] studied the effects of thermal radiation on the boundary layer flow of a Jeffery fluid over an exponentially stretching sheet. Ishak [9] studied boundary layer flow due to an exponentially stretching sheet with radiation effect. Mukhopadhyay and Gorla [10] analyzed the effects of partial slip on flow past an exponentially stretching sheet. Recently, Kirankumar et al. [11] worked on radiation and viscous dissipation effects on MHD heat transfer flow of Nano fluid over an exponentially stretching sheet in a porous medium. Goud et al. [12] worked on two dimensional boundary layer flows over an exponentially stretching sheet with thermal radiation and viscous dissipation.

Stratification is the division of fluid column into layers with different densities caused by differences in temperature or salinity or both. Thermal stratification has many applications in reservoirs, oceans, estuaries, salinity stratification in rivers, industrial food, etc. Researchers have investigated experimentally and numerically and reported on flow over a surface embedded in a thermally stratified medium in several studies due to its real life applications.

Mukhopadhyay [13] studied MHD boundary layer flow and heat transfer over an exponentially stretching sheet embedded in a thermally stratified medium subject to suction. It was reported that increase in stratification parameter in the absence of suction and also in the presence of suction corresponds to decrease in temperature profiles and increase in temperature gradient. Natural convection heat and mass transfer in a 
micropolar fluid with thermal and mass stratification was investigated by Srinvasacharya and Upendar [14].

Animasaun [15] investigated Casson fluid flow with variable viscosity and thermal conductivity along exponentially stretching sheet embedded in a thermally stratified medium with exponentially heat generation. He also reported that increased in the stratification parameter resulted in reduction of velocity and temperature profiles. MHD flow of Nano fluids over an exponentially stretching sheet embedded in a stratified medium with suction and radiation effects was investigated by Loganthan and Vimala [16]. He reported that increase in stratification parameter leads to decrease in the Nano fluid's temperature. Besthapu et al. [17] worked on mixed convection flow of thermally stratified MHD Nano fluid over an exponentially stretching surface with viscous dissipation effect. They reported that temperature and concentration profiles are reduced with an increase in thermally stratified parameter.

Fluid flow through porous layers is encountered in a host of natural and industrial processes, including the flow of oil, gas and water in reservoirs; groundwater flow in the porous earth sediments; flow of air into the lungs; blood flow through the lung tissues. Nandeppanavar et al. [18] investigated heat transfer through a porous medium over a stretching sheet with effect of viscous dissipation. Gireesha et al. [19], worked on boundary layer flow of dusty fluid over a radiating stretching surface embedded in a thermally stratified porous medium in the presence of uniform heat source. They reported that both temperature and velocity profiles decreases with increase in thermal stratification parameter

In this work, we extended the work of Mukhopadhyay [13] by considering the influence of radiation on MHD boundary layer flow over an exponentially stretching sheet embedded in a thermally stratified porous medium with heat source and suction/blowing. Using similarity transformations, the formulated governing system of partial differential equations is transformed into a system of non-linear ordinary differential equations and then solved numerically using shooting method along with fourth order Runge-Kutta technique. The effect of the controlling parameters on the fluid velocity and temperature distributions have been demonstrated graphically and discussed. A comparative study is also presented.

\section{Mathematical Formulation}

In this analysis, consider a steady, laminar, two dimensional, incompressible viscous 
electrically conducting fluid flow over a vertical exponentially stretching sheet embedded in a thermally stratified porous medium in the presence of suction and radiation effect coinciding with the plane $y=0$. The schematic illustration of the physical model is shown in Figure 1. The flow is confined to $y>0$. Two equal and opposite forces are applied along the x-axis so that the wall is stretched keeping the origin fixed (Fig. 1). The motion of the fluid is induced by the stretching property of the sheet. The porous medium is assumed to be diaphanous and in thermal equilibrium with the fluid. The wall conditions and the free stream temperature embedded in a thermally stratified medium are stated in Mukhopadyay [13].

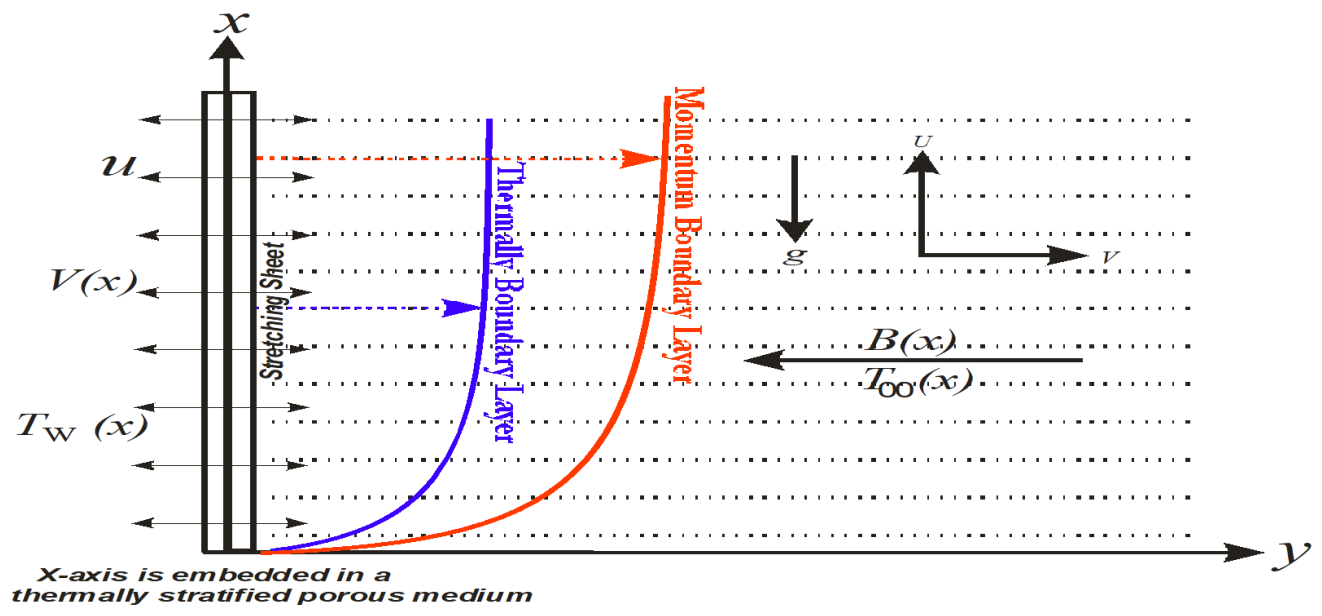

Figure 1. Sketch of physical problem.

A variable magnetic field $B(x)=B_{0} e^{\frac{x}{2 L}}$ of constant intensity $B_{0}$ is applied normal to the sheet. The sheet is of temperature $T_{w}(x)$ and is embedded in a thermally stratified porous medium of variable ambient temperature $T_{\infty}(x)$, where $T_{w}(x)>T_{\infty}(x)$. It is assumed that $T_{w}(x)=T_{0}+b e^{\frac{x}{2 L}}$ and $T_{\infty}(x)=T_{0}+c e^{\frac{x}{2 L}}$, where $T_{0}$ is the reference temperature, $b>0, c \geq 0$ are constants.

Since the fluid pressure is constant throughout the boundary layer, it is assumed that the induced magnetic field is small, hence it is neglected. Under the above assumptions, the boundary layer equations governing the flow and heat transfer of a viscous incompressible fluid can be written as:

$$
\frac{\partial u}{\partial x}+\frac{\partial v}{\partial y}=0
$$




$$
\begin{aligned}
& u \frac{\partial u}{\partial x}+v \frac{\partial u}{\partial y}=v \frac{\partial^{2} u}{\partial y^{2}}+g \beta\left(T-T_{\infty}\right)-\frac{\sigma B^{2}}{\rho} u-\frac{v}{K^{*}} u \\
& u \frac{\partial T}{\partial x}+v \frac{\partial T}{\partial y}=\frac{k}{\rho C_{p}} \frac{\partial^{2} T}{\partial y^{2}}+\frac{Q_{0}}{\rho C_{p}}\left(T-T_{\infty}\right)-\frac{1}{\rho C_{p}} \frac{\partial q_{r}}{\partial y}
\end{aligned}
$$

$(u, v)$ are the velocity components, $v$ is kinematic viscosity, $\rho$ is fluid density, $\mu$ is coefficient of fluid viscosity, $C_{p}$ is specific heat at constant pressure, $k$ is thermal conductivity of the fluid, $g$ is acceleration due to gravity, $\beta$ is volumetric coefficient of thermal expansion, $Q_{0}$ is dimensional heat generation, $K^{*}$ is permeability coefficient of porous medium, $\sigma$ is electrical conductivity, $q_{r}$ is radiative heat flux, $T$ is temperature.

Using Rosseland approximation for radiation,

$$
q_{r}=\frac{4 \sigma^{*}}{3 k^{*}} \frac{\partial T^{4}}{\partial y}
$$

where $\sigma^{*}=$ Stefan-Boltzmann constant and $k^{*}=$ absorption coefficient. By assuming that the temperature difference within the flow is such that $T^{4}$ may be expanded in a Taylor series and expanding $T^{4}$ about $T_{\infty}$ and neglecting higher orders we have:

$$
T^{4} \cong 4 T_{\infty}^{3} T-3 T_{\infty}^{3} \text {. }
$$

Substituting the partial derivative with respect to $T$ of equation (5) in equation (4) the rate of change of radiative heat flux in the $y$-axis direction becomes

$$
\frac{\partial q_{r}}{\partial y}=-\frac{16 \sigma^{*} T_{\infty}^{3}}{3 k^{*}} \frac{\partial^{2} T}{\partial y^{2}}
$$

Therefore equation (3) becomes;

$$
u \frac{\partial T}{\partial x}+v \frac{\partial T}{\partial y}=\frac{k}{\rho C_{p}} \frac{\partial^{2} T}{\partial y^{2}}+\frac{16 \sigma^{*} T_{\infty}^{3}}{3 k^{*}} \frac{\partial^{2} T}{\partial y^{2}}+\frac{Q_{0}}{\rho C_{p}}\left(T-T_{\infty}\right) .
$$

\subsection{Boundary conditions}

The appropriate boundary conditions for the problem are given by

$$
\begin{gathered}
u=U, v=-V(x), T=T_{w}(x) \quad \text { at } \quad y=0 . \\
u \rightarrow 0, \quad T \rightarrow T_{\infty} \quad \text { as } y \rightarrow \infty .
\end{gathered}
$$

Here $U=U_{0} e^{\frac{x}{L}}$ is the stretching velocity, $U_{0}$ is reference velocity $V(x)>0$ is velocity of suction and $V(x)<0$ is velocity of blowing, $V(x)=V_{0} e^{\frac{x}{2 L}}$ is considered a special type of velocity at the wall, $V_{0}$ is the initial strength of suction. Also, it is obvious that $\left(T_{w}-T_{0}\right)=b e^{\frac{x}{2 L}}$ and $\left(T_{\infty}-T_{0}\right)=c e^{\frac{x}{2 L}}$. 


\subsection{Similarity transformation}

Now introduce the stream function $\varphi(x, y)$ define by

$$
u=\frac{\partial \varphi}{\partial y} \quad v=-\frac{\partial \varphi}{\partial x}
$$

These automatically satisfy continuity equation. Next introduce the similarity transformations;

$$
\begin{gathered}
\varphi=\sqrt{2 v L U_{0}} f(\eta) e^{\frac{x}{2 L}}, \eta=y \sqrt{\frac{U_{0}}{2 v L}} e^{\frac{x}{2 L}}, \quad \theta(\eta)=\frac{T-T_{\infty}}{T_{w}-T_{0}}, \\
u=U_{0} e^{\frac{x}{L}} f^{\prime}(\eta), v=-\sqrt{\frac{v U_{0}}{2 L}} e^{\frac{x}{2 L}}\left\{f(\eta)+\eta f^{\prime}(\eta)\right\} .
\end{gathered}
$$

Substituting equations (9) and (10) into equations (2) to (4), the governing equations reduce to:

$$
\begin{gathered}
f^{\prime \prime \prime}+f f^{\prime \prime}-2 f^{2}+G_{m r} \theta-(M+K) f^{\prime}=0 \\
\left(1+\frac{4}{3} R\right) \theta^{\prime \prime}+P_{r}\left(f \theta^{\prime}-f^{\prime} \theta\right)+2 Q P_{r} \theta-P_{r} S t f^{\prime}=0 .
\end{gathered}
$$

The boundary conditions take the following forms

$$
\begin{array}{rlrlrl}
f^{\prime} & =1, & f=S \quad \theta=1-S t & \text { at } \eta=0 \\
\text { and } & f^{\prime} & \rightarrow 0, & \theta & \rightarrow 0 & \text { as } \quad \eta \rightarrow \infty .
\end{array}
$$

Here, primes denote differentiation with respect to the similarity variable $\eta$ and the dimensionless parameters are as follows:

$$
\begin{aligned}
& M=\frac{2 L \sigma B_{0}^{2}}{\rho U_{0}} \text { is the magnetic parameter; } \\
& G_{m r}=\frac{g \beta\left(T_{w}-T_{\infty}\right) 2 L \theta}{U_{0}^{2} e^{\frac{2 x}{L}}} \text { is the modified Grashof parameter; } \\
& K=\frac{2 v L}{K^{*} U_{0} e^{\frac{x}{L}}} \text { is the permeability parameter; } \\
& S t=C / b \text { is the stratification parameter; } \\
& S=\frac{V_{0}}{\sqrt{\frac{v U_{0}}{2 L}}>0(\text { or }<0) \text { is suction (or blowing)parameter; }} \\
& Q=\frac{Q_{0} L}{u_{0} e^{\frac{x}{L}} \rho C_{p}} \text { is the heat source; }
\end{aligned}
$$




$$
\begin{aligned}
& P_{r}=\mu /{ }_{k} \text { is the Prandtl number; } \\
& R=\frac{4 \sigma^{*} T_{\infty}^{3}}{k k^{*}} \text { is radiation parameter. }
\end{aligned}
$$

$S t>0$ implies a stably stratified environment, while $S t=0$ corresponds to an unstratified environment. For practical applications, the major physical quantities of interest are the local skin friction coefficient and Nusselt number. The physical quantity of interest is the wall skin friction coefficient $C_{f}$ defined as:

$$
C_{f}=\frac{\mu \tau_{w}}{\rho U_{0}^{2} e^{\frac{2 x}{L}}},
$$

where $\tau_{w}$ is known as the shear stress or the skin friction along the stretching sheet is given as:

$$
\begin{gathered}
\tau_{w}=\left.\frac{\partial U}{\partial y}\right|_{y=0} \\
f^{\prime \prime}=\sqrt{2} e^{\frac{x}{2 L}} C_{f} \sqrt{R e} .
\end{gathered}
$$

Another physical quantity of interest is the local Nusselt number $N u$, which is defined as

$$
N u=\frac{L q_{w}}{k\left(T_{w}-T_{0}\right)},
$$

where $q_{w}$ is known as heat flux from the sheet given as

$$
\begin{aligned}
& q_{w}=-\left.k \frac{\partial T}{\partial y}\right|_{y=0} \\
& -\theta^{\prime}=\sqrt{\frac{2}{R e}} \frac{N u}{e^{\frac{x}{2 L}}} .
\end{aligned}
$$

Here local Reynold number is given as

$$
R e=\frac{U_{0} x}{v} .
$$

\section{Numerical Solution}

We can notice that the set of equations (10) and (11) can be solved numerically using shooting method along with fourth order Runge-Kutta technique. Firstly, the non-linear differential equations (10) and (11) are decomposed into a system of first order differential equations. The governing dimensionless coupled higher order non-linear differential equations as well as the boundary conditions are transformed to system of 
first order differential equations. Using shooting method, the missing values are required but no such values are given after the boundary conditions were non-dimensioned. Suitable guessed values are chosen and the integration is carried out. The calculated values for $f^{\prime}$ and $\theta$ at $\eta_{\infty}=10$ are compared with the boundary condition. The better estimation for guess 1 and guess 2 are obtained, IVP are solved by using the fourth order Runge-Kutta technique with step size $h=0.1$. The above procedure is repeated until result up to desired degree of accuracy $10^{-5}$ is obtained. From the numerical computation, guess 1 is proportional to the skin-friction coefficient $f^{\prime \prime}(0)$ and guess 2 is proportional to Nusselt number $-\theta^{\prime}(0)$. The results obtained are presented in tables and MATLAB was used to plot the graphs and the main features are analyzed and discussed.

\section{Results and Discussion}

The influence of the dimensionless governing parameters namely: Magnetic parameter $(M)$, local modified Grashof related parameter $\left(G_{r m}\right)$, Thermal radiation parameter $(R)$, Prandtl number $\left(P_{r}\right)$, Heat source $(Q)$, Suction parameter $(S)$, Permeable parameter $(K)$ and Stratification parameter $\left(S_{t}\right)$ on the velocity and temperature distribution profiles are analyzed numerically using the method mentioned in the previous section. Numerical results are displayed with the help of graphs and table. Numerical values were plotted into graphs using MATLAB varying the fluid parameters with basic at $R=0.1, S_{t}=0.8, M=0.2, P_{r}=0.72, S=0.3, G_{r m}=1.0, K=1.0$ and $Q=4$.

To check the accuracy of the numerical method use, the velocity profile verses boundary layer coordinate $\eta$ of the present result was compared to the velocity profile put forward by Mukhopadhyay [13] when $R=Q=G_{r m}=K=0$ as shown in Figure 2(a) and 2(b). There was excellent agreement between the two plotted graphs. Therefore, we are confident that our results are accurate. 


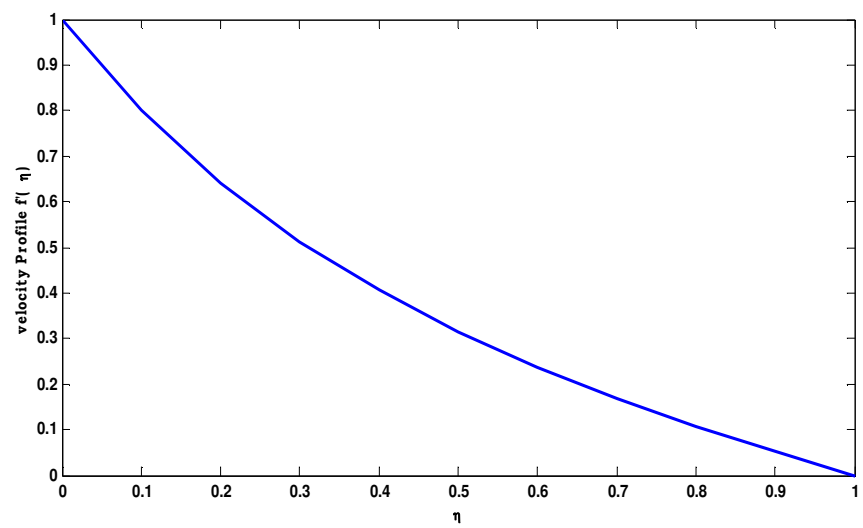

(a)

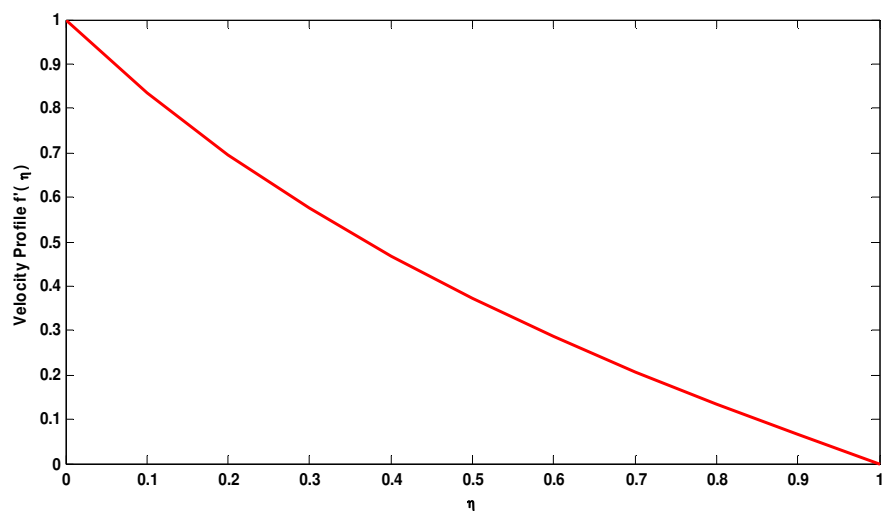

(b)

Figure 2. (a) Velocity profile of present work. (b) velocity profile of [13].

Figure 3 shows the variations in velocity profile of fluid in response to changes in the values of thermal stratification parameter $\left(S_{t}\right)$. It is observed that as the values of stratification parameter increases, the velocity decreases. 


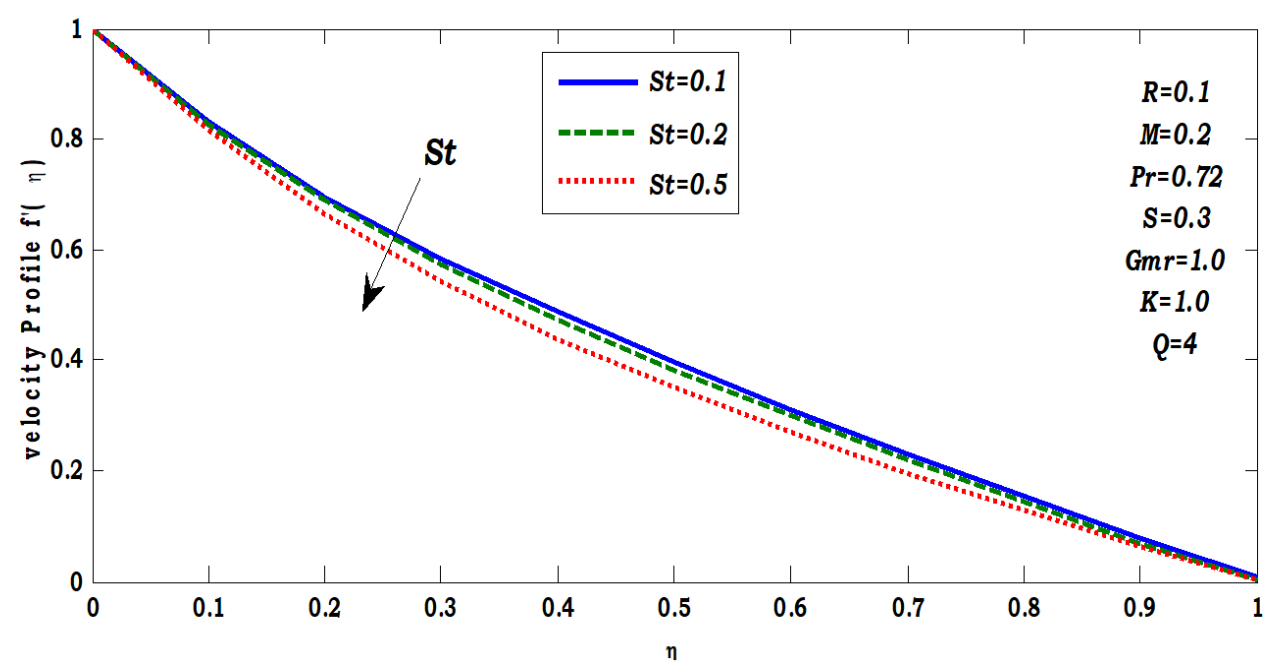

Figure 3. Influence of $\left(S_{t}\right)$ on velocity profile.

Figure 4 presents the influence of variation of magnetic parameter $(M)$ on velocity profiles of fluid. It is seen that the velocity decreases as the magnetic parameter $(M)$ values increases. This is due to the fact that increase in magnetic parameter induces a force called Lorentz force, which opposes fluid flow thereby reducing velocity.

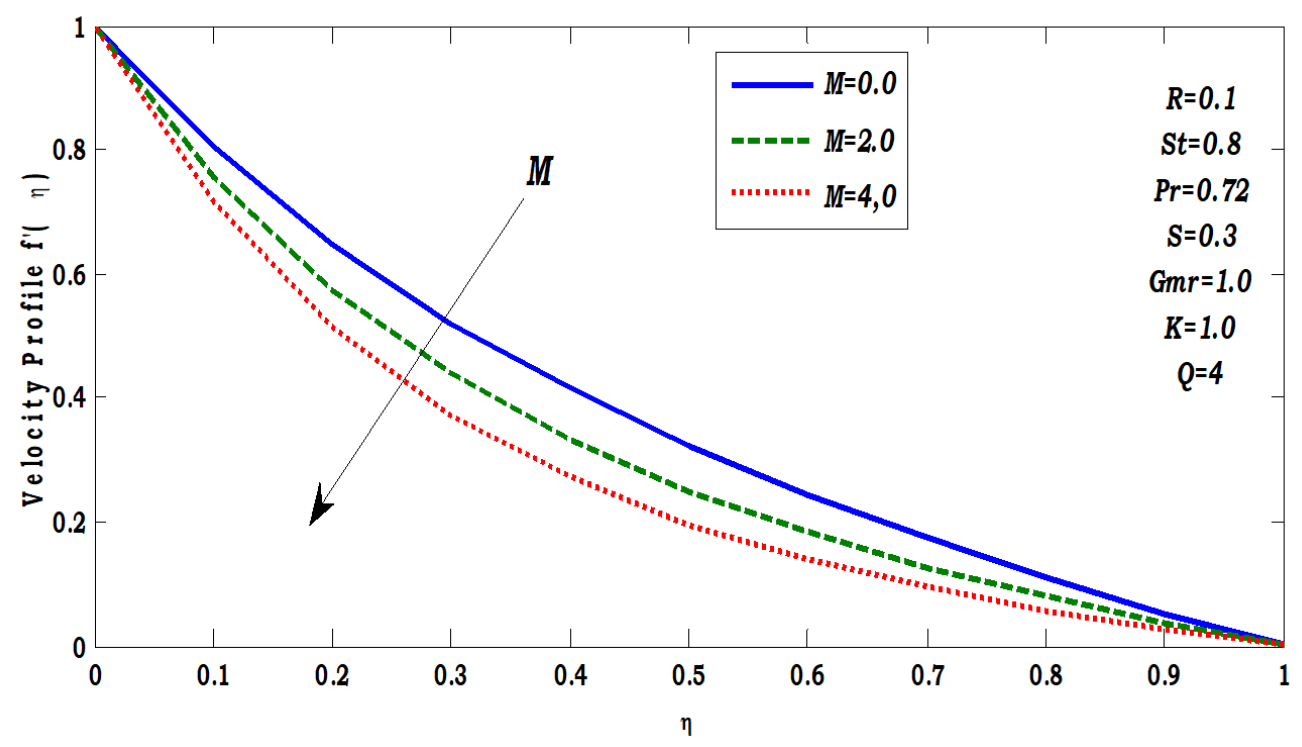

Figure 4. Influence of $(M)$ on velocity profile. 
Figure 5 shows the influence of permeable parameter $(K)$ on velocity profile. It is observed that as the permeable parameter $(K)$ values increases, there is reduction in the velocity. This is as a result of increase in permeable parameter $(K)$ increases the resistance to the fluid motion.

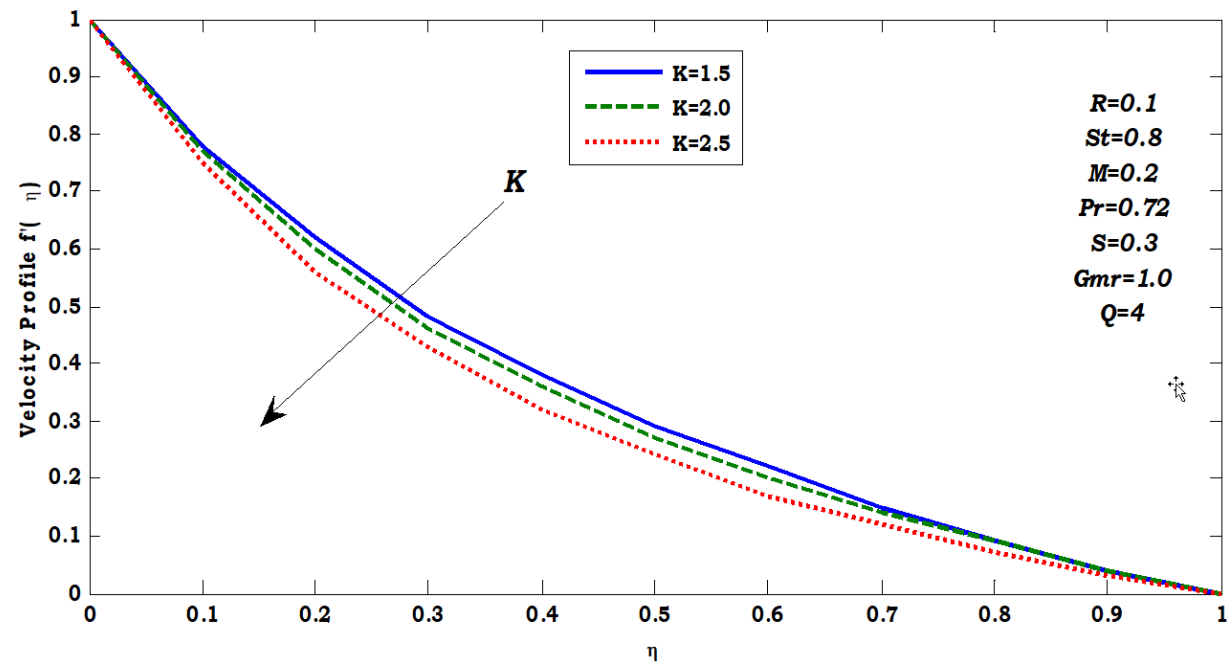

Figure 5. Influence of $(K)$ on velocity profile.

Figure 6 depicts the influence of suction parameter $(S)$ on dimensionless velocity profile. It is observed that increase in the values of suction parameter brings about decrease in the velocity distribution.

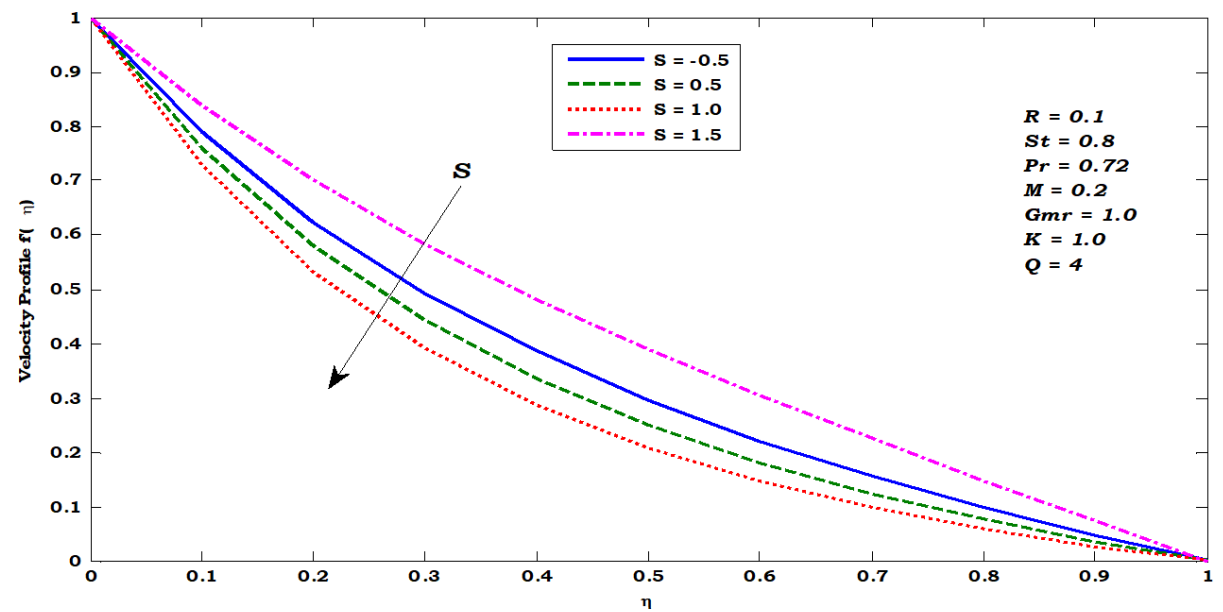

Figure 6. Influence of $(S)$ on velocity profile. 
Figure 7 illustrates influence of different values of Prandtl number on dimensionless velocity. The graph shows that increasing the values of Prandtl number results in a decreasing velocity.

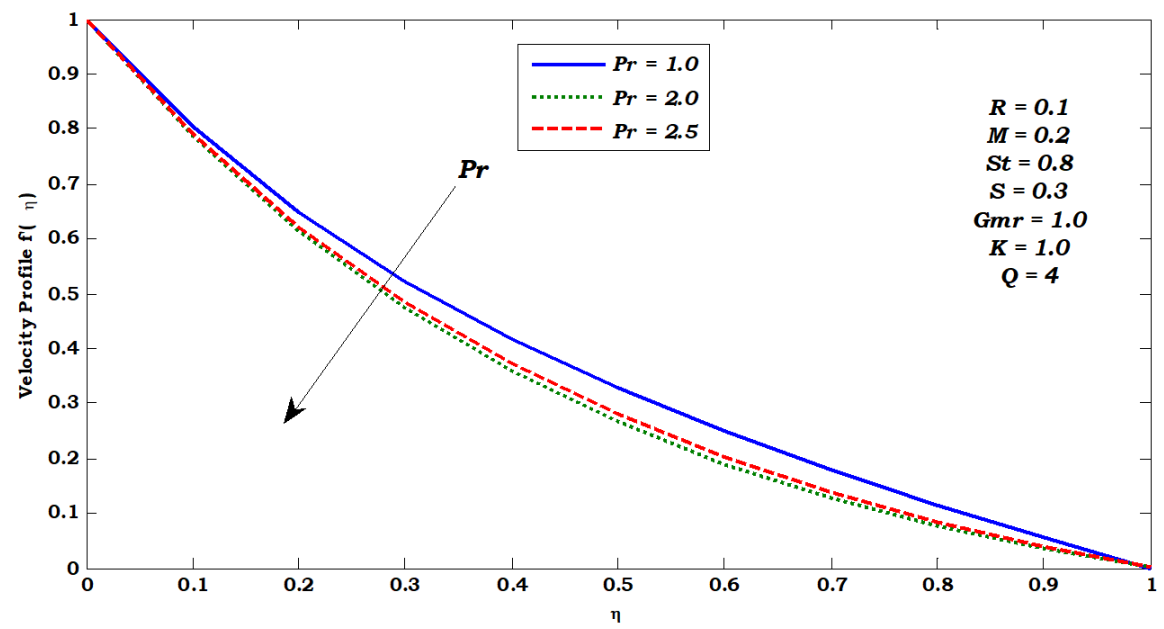

Figure 7. Influence of $(\mathrm{Pr})$ on velocity profile.

Figure 8 shows the influence of stratification parameter $\left(S_{t}\right)$ on temperature of fluid. It is observed that as the values of stratification parameter increases, the temperature profile decreases. This is due to the fact that an increase in stratification parameter means increase in free-stream temperature or decrease in surface temperature. Thermal boundary layer thickness also decreases with increase in stratification values.

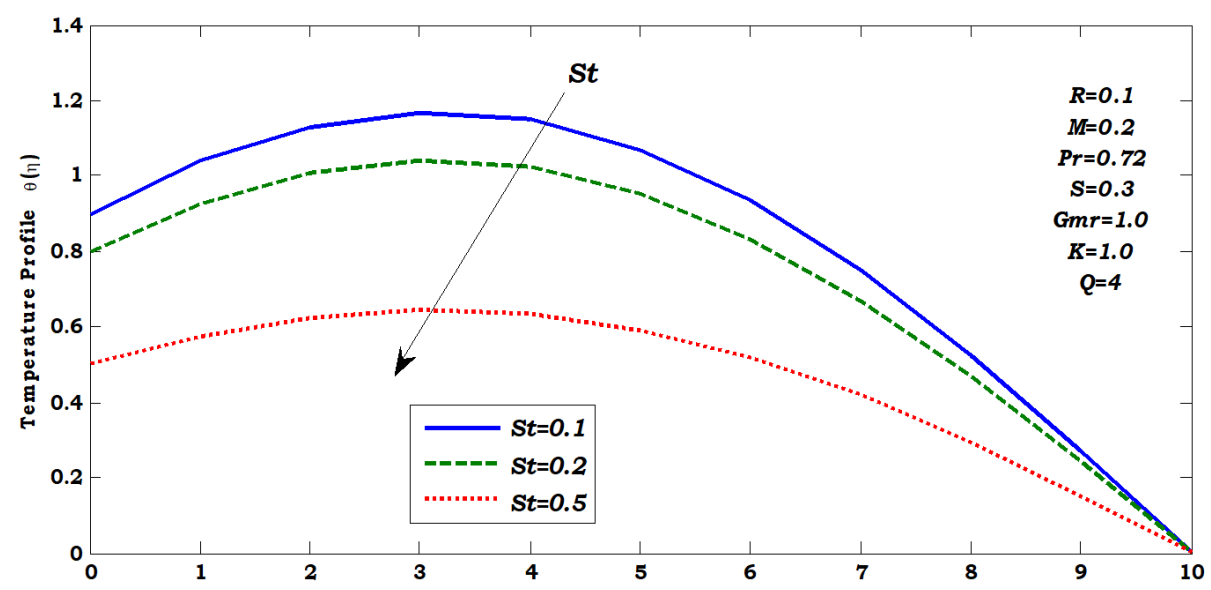

Figure 8. Influence of $(S t)$ on temperature profile. 
Figure 9 presents the influence of variation of heat source parameter on temperature profiles of fluid. It is seen that the temperature increases with increase in the values of heat source parameter and vice versa. This is due to the fact that additional energy was generated and this brought about the increase in thickness of thermal boundary layers.

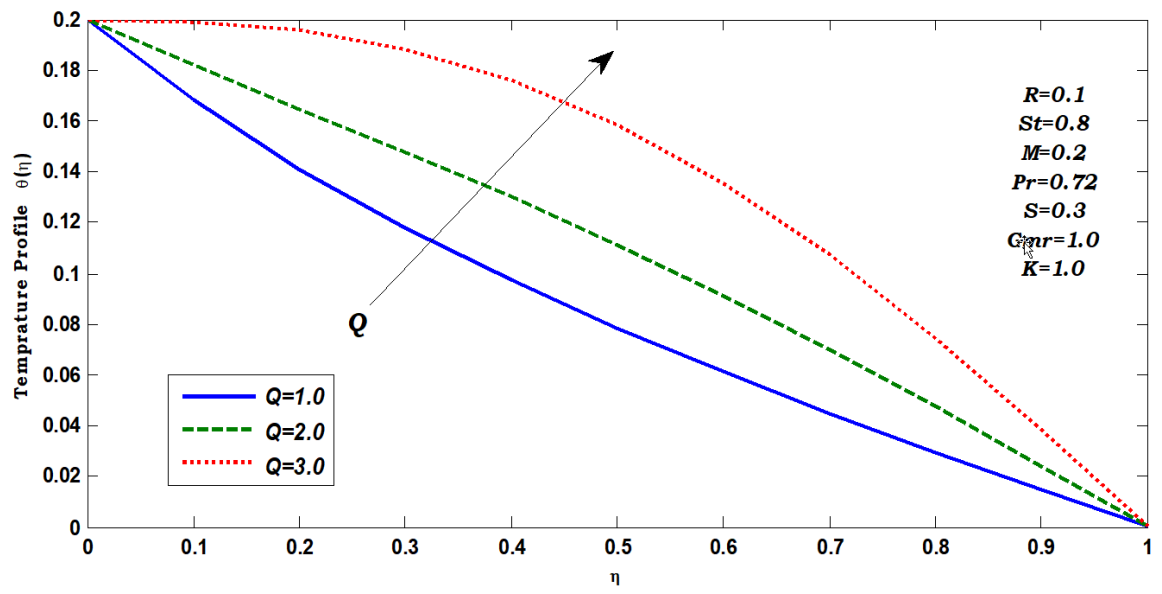

Figure 9. Influence of Heat $(Q)$ on temperature profile.

Figure 10 depicts the influence of magnetic parameter $(M)$ on temperature distribution of fluid. As a result of the induced Lorentz force caused by increase in magnetic parameter values, fluid flowed is slowed down and hence increases in the temperature of the fluid.

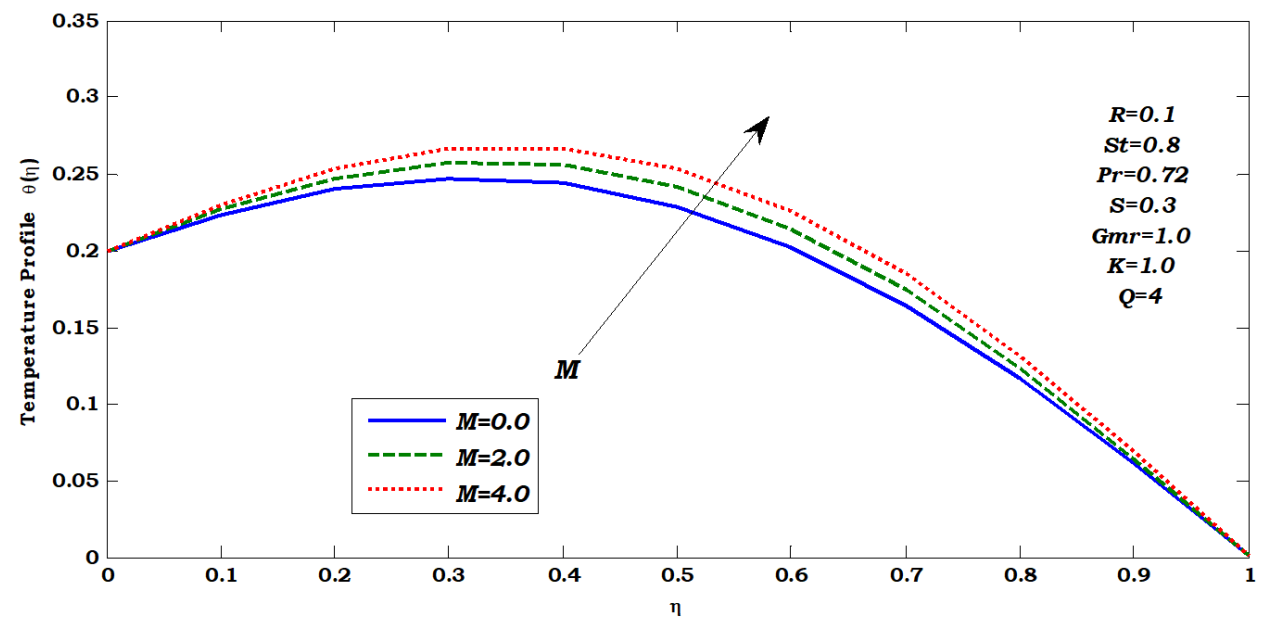

Figure 10. Influence of $(M)$ on temperature profile. 
Figure 11 explains the influence of permeable parameter $(K)$ on the dimensionless temperature distribution of fluid. Increase in the values of permeable parameter brings about increase in the temperature of the fluid.

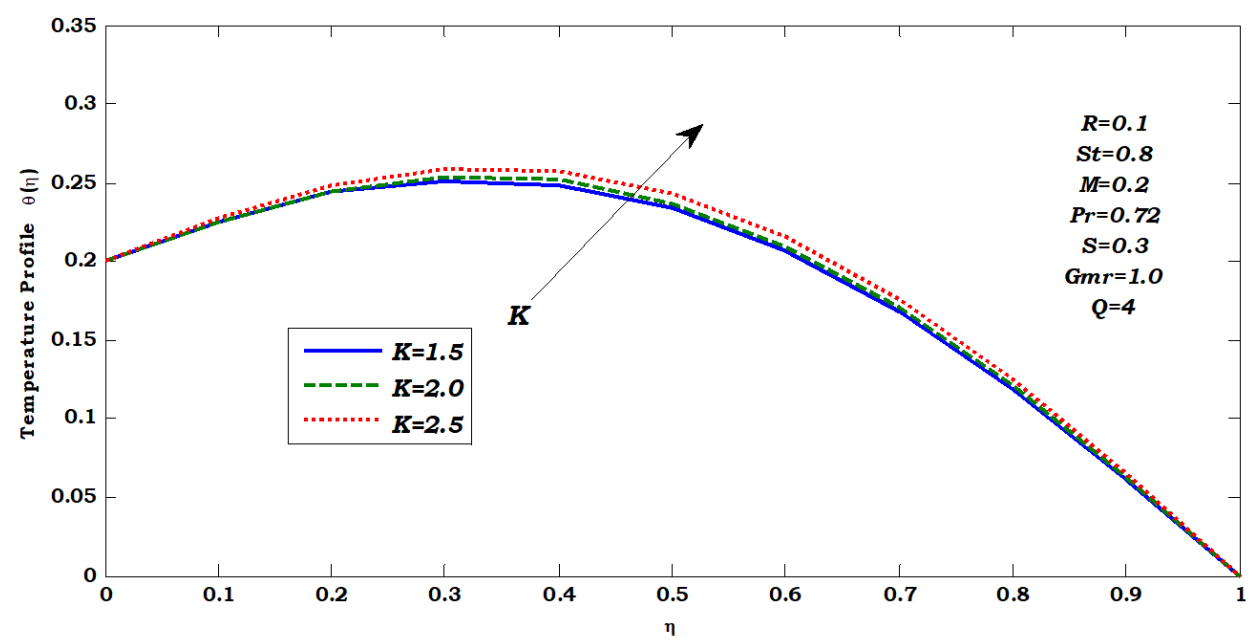

Figure 11. Influence of $(K)$ on temperature profile.

The influence of suction parameter on temperature is seen in Figure 12. The figure illustrates that the temperature of the fluid decreases as the suction parameter $(S)$ values increases.

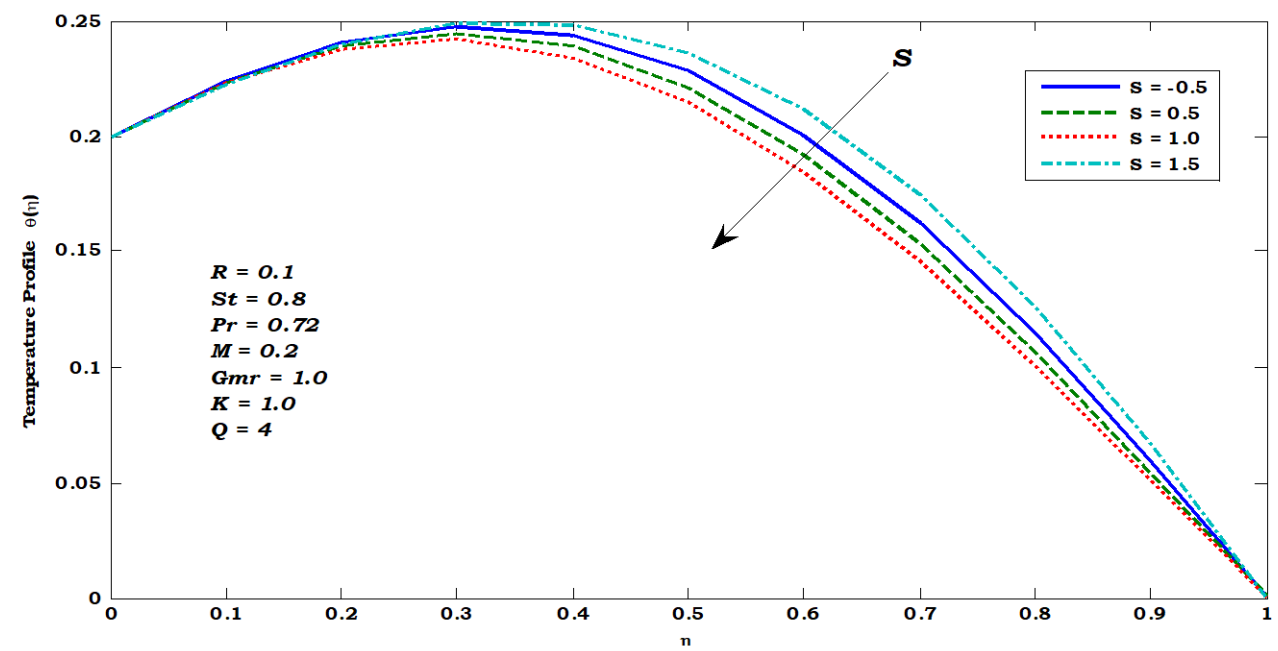

Figure 12. Influence of $(S)$ on temperature profile. 
The influence of the radiation parameter $(R)$ on the temperature of the fluid is presented in Figure 13. It can be easily seen that the temperature and the thermal boundary layer thickness decreases with increasing radiation parameter values.

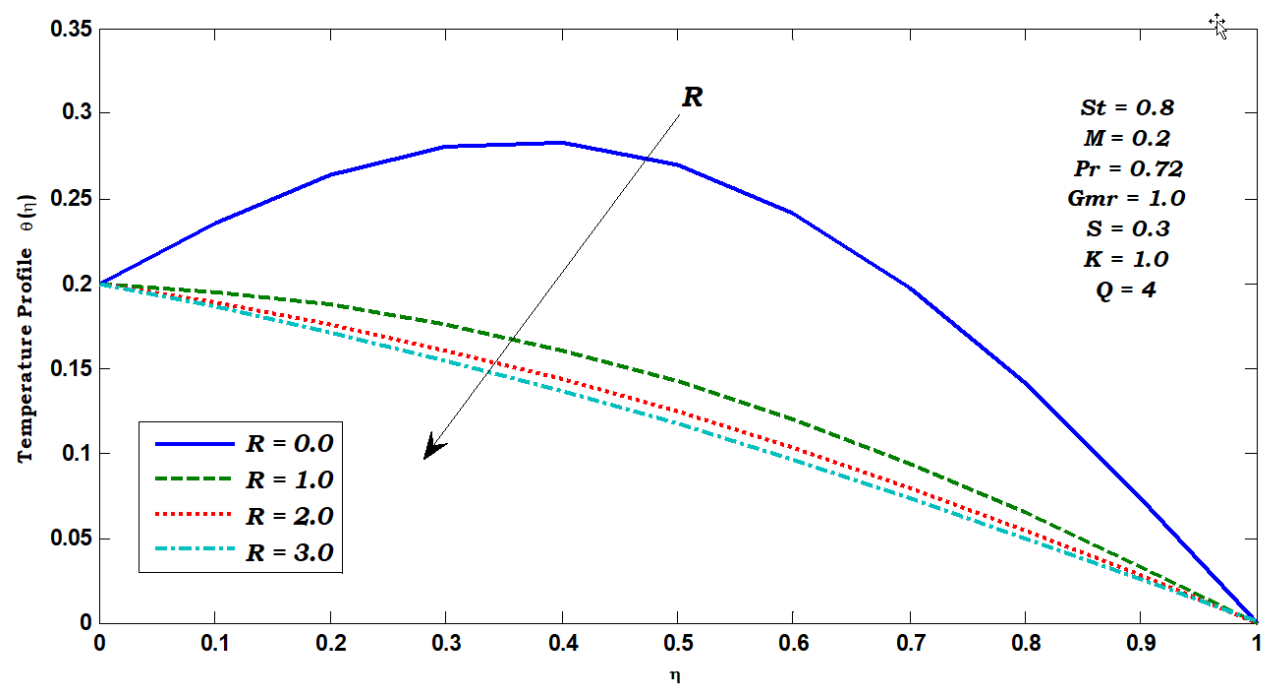

Figure 13. Influence of $(R)$ on temperature profile.

Table 1 represents values of skin-friction coefficient and Nusselt number for various values of: Magnetic parameter $(M)$, Thermal radiation parameter $(R)$, Heat source $(Q)$, Suction parameter $(S)$, Permeable parameter $(K)$ and Stratification parameter $\left(S_{t}\right)$ with constant values for local modified Grashof related parameter $\left(G_{r m}\right)$ and Prandtl number $\left(P_{r}\right)$.

Table 1. The numerical values of $f^{\prime \prime}(0)$ and $-\theta^{\prime}(0)$ different values $M, R, Q, S, K$ and $S_{t}$ with $P_{r}=0.72$ and $G_{r m}=1.0$.

\begin{tabular}{|c|c|c|c|c|c|c|c|}
\hline $\boldsymbol{S}_{\boldsymbol{t}}$ & $\boldsymbol{R}$ & $\boldsymbol{K}$ & $\boldsymbol{Q}$ & $\boldsymbol{M}$ & $\boldsymbol{S}$ & $\boldsymbol{f}^{\prime \prime}(\mathbf{0})$ & $-\boldsymbol{\theta}^{\prime}(\mathbf{0})$ \\
\hline 0.1 & 0.1 & 1.0 & 4 & 0.2 & 0.3 & -0.6453 & -2.5900 \\
\hline 0.2 & 0.1 & 1.0 & 4 & 0.2 & 0.3 & -0.6266 & -2.3152 \\
\hline 0.5 & 0.1 & 1.0 & 4 & 0.2 & 0.3 & -0.5397 & -1.4652 \\
\hline 0.8 & 1 & 1.0 & 4 & 0.2 & 0.3 & -0.4300 & -0.3277 \\
\hline 0.8 & 2 & 1.0 & 4 & 0.2 & 0.3 & -0.4255 & -0.2694 \\
\hline
\end{tabular}




\begin{tabular}{|c|c|c|c|c|c|c|c|}
\hline 0.8 & 3 & 1.0 & 4 & 0.2 & 0.3 & -0.4237 & -0.2458 \\
\hline 0.8 & 0.1 & 1.5 & 4 & 0.2 & 0.3 & -0.4139 & -0.6173 \\
\hline 0.8 & 0.1 & 2.0 & 4 & 0.2 & 0.3 & -0.3787 & -0.6289 \\
\hline 0.8 & 0.1 & 2.5 & 4 & 0.2 & 0.3 & -0.3183 & -0.6503 \\
\hline 0.8 & 0.1 & 1.0 & 1 & 0.2 & 0.3 & -0.4141 & -0.1370 \\
\hline 0.8 & 0.1 & 1.0 & 2 & 0.2 & 0.3 & -0.4221 & -0.2345 \\
\hline 0.8 & 0.1 & 1.0 & 3 & 0.2 & 0.3 & -0.4342 & -0.3783 \\
\hline 0.8 & 0.1 & 1.0 & 4 & 0.0 & 0.3 & -0.4691 & -0.6003 \\
\hline 0.8 & 0.1 & 1.0 & 4 & 2.0 & 0.3 & -0.3294 & -0.6449 \\
\hline 0.8 & 0.1 & 1.0 & 4 & 4.0 & 0.3 & -0.2364 & -0.6830 \\
\hline 0.8 & 0.1 & 1.0 & 4 & 0.2 & 0.5 & -0.4005 & -0.5825 \\
\hline 0.8 & 0.1 & 0.1 & 4 & 0.2 & 1.0 & -0.2914 & -0.5255 \\
\hline
\end{tabular}

From Table 1, it is seen that the values of the skin-friction coefficient are always negative. Physically, negative sign of skin-friction implies that the stretching sheet exerts a drag force on the fluid that causes the movement of the fluid on the surface. Table 1 shows that increase in the stratification parameter, radiation parameter and suction parameter leads to increase in skin-friction coefficient and Nusselt number, whereas, increase in the heat source parameter brings about decrease in both skin-friction coefficient and Nusselt number. Increase in magnetic and permeable parameters brings about increase in skin-friction coefficient and decrease in Nusselt number.

\section{Conclusion}

Two dimensional electrically conducting MHD flow was discussed in this research work. Similarity transformations are used for the conversion of nonlinear partial differential equations to nonlinear ordinary differential equations. Numerical solutions are carried out using shooting method along with fourth order Runge-Kutta's technique and graphical results of velocity profile are compared with previous work as a limiting case, and excellent accuracy is achieved with those results. The effects of various controlling parameters on the flow and heat transfer are observed from the graphs and are summarized as follows: 
* Increase in the values of the stratification parameter $\left(S_{t}\right)$ result in decrease of both the velocity and temperature distribution of the fluid.

* Increase in the values of permeable parameter $(Q)$ leads to increase in temperature and decrease in velocity distribution of the fluid.

* Thermal boundary layer thickness increases with an increase in the values of magnetic parameter $(M)$.

* The velocity and temperature distribution of the fluid decreases with increasing values of suction parameter $(S)$.

* The thickness of momentum boundary layer decreases with an increase in the value of magnetic parameter $(M)$.

* The skin-friction coefficient and Nusselt number is enhanced with increasing values of the stratification parameter $\left(S_{t}\right)$, radiation parameter $(R)$ and suction parameter $(S)$.

\section{References}

[1] B.C. Sakiadis, Boundary-layer behaviour on continuous solid surfaces: I. Boundary-layer equations for two-dimensional and axisymmetric flow, AIChE Journal 7 (1961), 26-28. https://doi.org/10.1002/aic.690070108

[2] L.J. Crane, Flow past a stretching plate, Z. Angew. Math. Phys. 21 (1970), 645-647. https://doi.org/10.1007/BF01587695

[3] P.S. Gupta and A.S. Gupta, Heat and mass transfer on a stretching sheet with suction or blowing, Can. J. Chem. Eng. 55 (1977), 744-746.

https://doi.org/10.1002/cjce.5450550619

[4] E.M.A. Elbashbeshy, Heat transfer over an exponentially stretching continuous surface with suction, Arch. Mech. 53 (2001), 643-651.

[5] B. Bidin, and R. Nazar, Numerical solution of the boundary layer flow over an exponentially stretching sheet with thermal radiation, Eur. J. Sci. Res. 33(4) (2009), 710717.

[6] M. Sajid and T. Hayat, Influence of thermal radiation on the boundary layer flow due to an exponentially stretching continuous sheet, Int. Commun. Heat Mass Transfer 35 (2008), 347-356. https://doi.org/10.1016/j.icheatmasstransfer.2007.08.006

[7] A. S. Idowu and S. Usman, Influence of thermal radiation on magnetohydrodynamic 
(MHD) boundary layer flow of a viscous fluid over an exponentially stretching sheet, American Journal of Engineering Research (AJER) 4 (2015), 70-80.

[8] S. Nadeem, S. Zaheer and T. Fang, Effects of thermal radiation on the boundary layer flow of a Jeffrey fluid over an exponentially stretching surface, Numer. Algor. 57 (2011), 187-205. https://doi.org/10.1007/s11075-010-9423-8

[9] A. Ishak, MHD boundary layer flow due to an exponentially stretching sheet with radiation effect, Sains Malaysiana 40 (2011), 391-395.

[10] S. Mukhopadhyay and R.S.R. Gorla, Effects of partial slip on boundary layer flow past a permeable exponential stretching sheet in presence of thermal radiation, Heat Mass Transfer 48 (2012), 1773-1781. https://doi.org/10.1007/s00231-012-1024-8

[11] R.V.M.S.S. Kirankumar, P. Durga Prasad, V. Nagendramma, A. Leelaratnam and S.V.K. Varma, Radiation and viscous dissipation effects on MHD heat transfer flow of nanofluid over an exponentially stretching sheet in a porous medium, International Journal of Pure and Applied Mathematics 113(7) (2017), 155-163.

[12] B.S. Goud, R.K. Jilugu, R. Gugulothu and K. Shivakumar, Effects of the thermal radiation on the boundary layer flow over an exponentially stretching sheet in the presence of viscous dissipation, International Journal of Modern Engineering and Research Technology 6 (2019), 240-245.

[13] S. Mukhopadhyay, MHD boundary layer flow and heat transfer over an exponentially stretching sheet embedded in a thermally stratified medium, Alexandria Engineering Journal 52 (2013), 259-265. https://doi.org/10.1016/j.aej.2013.02.003

[14] D. Srinivasacharya and M. Upendar, Effect of double stratification on MHD free convection in a micropolar fluid, Journal of the Egyptian Mathematical Society 21(3) (2013), 370-378. https://doi.org/10.1016/j.joems.2013.02.006

[15] I. L. Animasaun, Casson fluid flow with variable viscosity and thermal conductivity along exponentially stretching sheet embedded in a thermally stratified medium with exponentially heat generation, Journal of Heat and Mass Transfer Research 2(2) (2015), 63-78. https://dx.doi.org/10.22075/jhmtr.2015.346

[16] P. Loganthan and C. Vimala, MHD flow of Nanofluids over an exponentially stretching sheet embedded in a stratified medium with suction and radiation effects, Journal of Applied Fluid Mechanics 8(1) (2015), 85-93. https://doi.org/10.36884/jafm.8.01.22556

[17] Prabhakar Besthapu, Rizwan Ul Haq, Shankar Bandari and Qasem M. Al-Mdallal, Mixed convection flow of thermally stratified MHD nanofluid over an exponentially stretching surface with viscous dissipation effect, Journal of the Taiwan Institute of Chemical Engineers 71 (2017), 307-314. https://doi.org/10.1016/j.jtice.2016.12.034 
[18] Mahantesh M. Nandeppanavar, A. Subhas and M.N. Siddalingappa, Heat transfer through a porous medium over a stretching sheet with effect of viscous dissipation, Chemical Engineering Communications 200(11) (2013), 1513-1529.

https://doi.org/10.1080/00986445.2012.749247

[19] B.J. Gireesha, P. Venkatesh, N.S. Shashikumar and B. Prasannakumara, Boundary layer flow of dusty fluid over a radiating stretching surface embedded in a thermally stratified porous medium in the presence of uniform heat source, Nonlinear Engineering 6(1) (2017), 31-41. https://doi.org/10.1515/nleng-2016-0058

This is an open access article distributed under the terms of the Creative Commons Attribution License (http://creativecommons.org/licenses/by/4.0/), which permits unrestricted, use, distribution and reproduction in any medium, or format for any purpose, even commercially provided the work is properly cited. 